\title{
INVESTIGATION OF THERMODYNAMIC PROPERTIES OF METAL THIN FILM BY STATISTICAL MOMENT METHOD
}

\author{
VU VAN HUNG \\ Vietnam Education Publishing House, \\ 81 Tran Hung Dao, Hanoi, Vietnam \\ DUONG DAI PHUONG \\ Hanoi National University of Education, \\ 136 Xuan Thuy Street, Hanoi, Vietnam \\ NGUYEN THI HOA \\ Fundamental Science Faculty, \\ University of Transport and Communications, \\ Lang Thuong, Dong Da, Hanoi, Vietnam \\ Received 28 September 2013 \\ Accepted for publication 20 December 2013
}

\begin{abstract}
The thermodynamic properties of the metal thin film with face-centered cubic structure at zero pressure are investigated using the statistical moment method (SMM), including the anharmonicity effects of thermal lattice vibrations. The Helmholtz free energy, linear thermal expansion coefficients, specific heats at the constant volume and those at the constant pressure, $C_{V}$ and $C_{p}$ are derived in closed analytic forms in terms of the power moments of the atomic displacements. Numerical calculations for thermodynamic quantities of Al, Au and $\mathrm{Ag}$ thin films are found to be in good agreement with those of the other theoretical results and experimental data.
\end{abstract}

\section{INTRODUCTION}

Materials in the form of thin films have become quite interesting for recent years. They have been found to show different physical, chemical, and mechanical properties from the corresponding bulk materials [1,2]. Properties, limitations and advantages obtained on thin films geometry have been widely studied, and they were found to depend on different factors: structure, size, film thickness, different substrates, ... [2,3]. Thin films are used in a vast range of applications such as microelectromechanical and nanoelectromechanical systems [4], sensors and electronic textiles [5], ...

Metal thin film is a common geometry and presents enormous scientific interests, mainly due to their attractive novel properties for technological applications $[4,5]$. In the early of the twentieth century, the theory of size-dependent effects in metal thin layers was further developed by various scientists [3]. For ultrathin metal layers both surface effects and quantum size effects must be considered [6]. A large number of experimental and theoretical studies have been carried out on thermal behavior of metal and nonmetal thin films $[7,8]$. 
Most of the previous theoretical studies, however, are concerned with the materials properties of metal thin film at low temperature, and temperature dependence of the thermodynamic quantities has not been studied in detail. The purpose of the present article is to investigate the temperature dependence and the thickness dependence of the thermodynamic properties of metal thin film with face-centered cubic structure using the analytic statistical moment method $(S M M)[9,10]$. The thermodynamic quantities are derived from the Helmholtz free energy, and the explicit expressions of the thermal lattice expansion coefficient, specific heats at constant volume and those at the constant pressure $C_{V}$ and $C_{p}$ are presented taking into account the anharmonicity effects of the thermal lattice vibrations. In the present study, the influence of surface and size effects on the thermodynamic properties have also been studied. We have compared the results of the present calculations with those of the previous theoretical calculations as well as with the available experimental results.

\section{THEORY}

\section{II.1. The anharmonic oscillations of the metal thin film}

Let us consider a metal free thin film including $n *$ layers with the film thickness $d$. Suppose that the thin film has been consisting of two surface layers atoms, two next surface layers atoms and $\left(n^{*}-4\right)$ internal layers atoms (Fig.1).

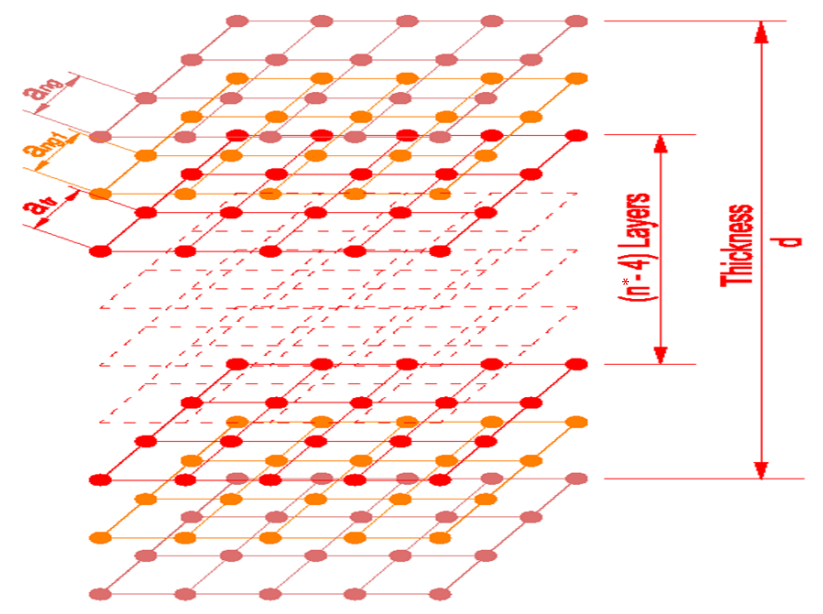

Fig. 1. The metal thin film.

First of all, we present the statistical moment method $(S M M)[9,10]$ formulation for the displacement of the surface layers atom of the thin film $\left\langle u_{i}^{n g}\right\rangle$, that is solution of equation

$$
\begin{aligned}
& \gamma_{n g} \theta^{2} \frac{d^{2}<u_{i}^{n g}>}{d p^{2}}+3 \gamma_{n g} \theta<u_{i}^{n g}>\frac{d<u_{i}^{n g}>}{d p}+\gamma_{n g}<u_{i}^{n g}>^{3}+k_{n g}<u_{i}^{n g}> \\
& +\gamma_{n g} \frac{\theta}{k_{n g}}\left(x_{n g} \operatorname{coth} x_{n g}-1\right)<u_{i}^{n g}>-p=0
\end{aligned}
$$


where

$$
\begin{gathered}
x_{n g}=\frac{\hbar \omega_{n g}}{2 \theta} ; \theta=k_{B} T ; k_{n g}=\frac{1}{2} \sum_{i}\left(\frac{\partial^{2} \varphi_{i o}^{n g}}{\partial u_{i \alpha}^{2}}\right)_{e q} \equiv m_{0} \omega_{n g}^{2}, \\
\gamma_{1 n g}=\frac{1}{48} \sum_{i}\left(\frac{\partial^{4} \varphi_{i o}^{n g}}{\partial u_{i \alpha}^{4}}\right)_{e q} ; \gamma_{2 n g}=\frac{6}{48} \sum_{i}\left(\frac{\partial^{4} \varphi_{i o}^{n g}}{\partial u_{i \beta}^{2} \partial u_{i \gamma}^{2}}\right)_{e q} \\
\gamma_{n g}=\frac{1}{12} \sum_{i}\left[\left(\frac{\partial^{4} \varphi_{i o}^{n g}}{\partial u_{i \alpha}^{4}}\right)_{e q}+6\left(\frac{\partial^{4} \varphi_{i o}^{n g}}{\partial u_{i \beta}^{2} \partial u_{i \gamma}^{2}}\right)_{e q}\right]=4\left(\gamma_{1 n g}+\gamma_{2 n g}\right) \\
\alpha, \beta, \gamma=x, y, z .
\end{gathered}
$$

Here, $k_{B}$ is the Boltzmann constant, $T$ is the absolute temperature, $m_{0}$ is the mass of atom at lattice node, $\omega_{n g}$ is the frequency of lattice vibration of surface layers atoms; $k_{n g} ; \gamma_{1 n g} ; \gamma_{2 n g} ; \gamma_{n g}$ are the parameters of crystal depending on the structure of crystal lattice and the interaction potential between atoms at node; $\varphi_{i 0}^{n g}$ is the effective interatomic potential between $0^{\text {th }}$ and $i^{\text {th }}$ surface layers atom; $u_{i x} ; u_{i y} ; u_{i z}$ are the displacement of $i^{\text {th }}$ atom from equilibrium position on direction $x, y, z$ respectively, and the subscript $e q$ indicates evaluation at equilibrium.

In determining the atomic displacement $\left\langle u_{i}^{n g}\right\rangle$, the symmetry property appropriate for cubic crystals is used

$$
<u_{i x}^{n g}>=<u_{i y}^{n g}>=<u_{i z}^{n g}>=<u_{i}^{n g}>.
$$

Then, the solutions of the nonlinear differential equation of Eq. (1) can be expanded in the power series of the supplemental force $p$ as

$$
<u_{i}^{n g}>=<u_{i}^{n g}>_{0}+A_{1} p+A_{2} p^{2} .
$$

Here, $<u_{i}^{n g}>_{0}$ is the average atomic displacement in the limit of zero of supplemental force $p$. Substituting the above solution of Eq. (6) into the original differential Eq. (1), one can get the coupled equations of the coefficients $A_{1}, A_{2}$, from which the solution of $<u_{i}^{n g}>_{0}$ is given as $[9,10]$

where

$$
<u_{i}^{n g}>_{0} \approx \sqrt{\frac{2 \gamma_{n g} \theta^{2}}{3 k_{n g}^{3}} A_{n g}}
$$

$$
A_{n g}=a_{1}^{n g}+\frac{\gamma_{n g}^{2} \theta^{2}}{k_{n g}^{4}} a_{2}^{n g}+\frac{\gamma_{n g}^{3} \theta^{3}}{k_{n g}^{6}} a_{3}^{n g}+\frac{\gamma_{n g}^{4} \theta^{4}}{k_{n g}^{8}} a_{4}^{n g}+\frac{\gamma_{n g}^{5} \theta^{5}}{k_{n g}^{10}} a_{5}^{n g}+\frac{\gamma_{n g}^{6} \theta^{6}}{k_{n g}^{12}} a_{6}^{n g},
$$

with $a_{\eta}^{n g}(\eta=1,2 \ldots, 6)$ are the values of parameters of crystal depending on the structure of crystal lattice $[9,10]$.

A similar derivation can be also done for next surface layers atoms and internal layers atoms of thin film. Their displacements are a solution of equations

$$
\begin{aligned}
& \gamma_{n g 1} \theta^{2} \frac{d^{2}<u_{i}^{n g 1}>}{d p^{2}}+3 \gamma_{n g 1} \theta<u_{i}^{n g 1}>\frac{d<u_{i}^{n g 1}>}{d p}+\gamma_{n g 1}<u_{i}^{n g 1}>^{3}+k_{n g 1}<u_{i}^{n g 1}> \\
& +\gamma_{n g 1} \frac{\theta}{k_{n g 1}}\left(x_{n g 1} \operatorname{coth} x_{n g 1}-1\right)<u_{i}^{n g 1}>-p=0,
\end{aligned}
$$




$$
\begin{aligned}
& \gamma_{t r} \theta^{2} \frac{d^{2}<u_{i}^{t r}>}{d p^{2}}+3 \gamma_{t r} \theta<u_{i}^{t r}>\frac{d<u_{i}^{t r}>}{d p}+\gamma_{t r}<u_{i}^{t r}>^{3}+k_{t r}<u_{i}^{t r}> \\
& +\gamma_{t r} \frac{\theta}{k_{t r}}\left(x_{t r} \operatorname{coth} x_{t r}-1\right)<u_{i}^{t r}>-p=0 .
\end{aligned}
$$

Thus, using the statistical moment method $[9,10]$, we can get power moments of the atomic displacement, including the anharmonicity effects of thermal lattice vibrations.

\section{II.2. Free energy of the metal thin film}

In the present research, the influence of the size effect on thermodynamic properties of the metal thin film is studied by introducing the surface energy contribution in the free energy of the system atoms. Following exactly the general formula in the SMM formulation $[9,10]$, one can get the free energy of the surface layers atoms as

$$
\begin{aligned}
& \Psi_{n g} \approx\left\{U_{0}^{n g}+3 N_{n g} \theta\left[x_{n g}+\ln \left(1-e^{-2 x_{n g}}\right)\right]\right\}+\frac{3 N_{n g} \theta^{2}}{k_{n g}^{2}}\left\{\gamma_{2 n g} X_{n g}^{2}-\frac{2 \gamma_{1 n g}}{3}\left(1+\frac{X_{n g}}{2}\right)\right\} \\
& +\frac{6 N_{n g} \theta^{3}}{k_{n g}^{4}}\left\{\frac{4}{3} \gamma_{2 n g}^{2}\left(1+\frac{X_{n g}}{2}\right) X_{n g}-2\left(\gamma_{1 n g}^{2}+2 \gamma_{1 n g} \gamma_{2 n g}\right)\left(1+\frac{X_{n g}}{2}\right)\left(1+X_{n g}\right)\right\},
\end{aligned}
$$

here $X_{n g}=x_{n g} \operatorname{coth} x_{n g}$.

The free energies of the next surface layers atoms and internal layers atoms have formula similar Eq. (11) and be noted $\Psi_{n g 1} ; \Psi_{t r}$, respectively.

The free energies in the harmonic approximation for surface layers, next surface layers atoms and internal layers atoms of thin film are given as

$$
\begin{aligned}
\Psi_{n g} & \approx 3 N_{n g}\left\{\frac{1}{6} u_{0}^{n g}+\theta\left[x_{n g}+\ln \left(1-e^{-2 x_{n g}}\right)\right]\right\}, \\
\Psi_{n g 1} & \approx 3 N_{n g 1}\left\{\frac{1}{6} u_{0}^{n g 1}+\theta\left[x_{n g 1}+\ln \left(1-e^{-2 x_{n g 1}}\right)\right]\right\}, \\
\Psi_{t r} & \approx 3 N_{t r}\left\{\frac{1}{6} u_{0}^{t r}+\theta\left[x_{t r}+\ln \left(1-e^{-2 x_{t r}}\right)\right]\right\},
\end{aligned}
$$

where

$$
\begin{aligned}
& u_{0}^{n g}=\sum_{i} \varphi_{i 0}^{n g}, u_{0}^{n g 1}=\sum_{i} \varphi_{i 0}^{n g 1}, u_{0}^{t r}=\sum_{i} \varphi_{i 0}^{t r}, \\
& U_{0}^{n g}=\frac{N_{n g}}{2} u_{0}^{n g}, U_{0}^{n g 1}=\frac{N_{n g 1}}{2} u_{0}^{n g 1}, U_{0}^{t r}=\frac{N_{t r}}{2} u_{0}^{t r} .
\end{aligned}
$$

Let us consider the system $N$ atoms consisting $n^{*}$ layers, the number atoms on each layer is the same as $N_{L}$. $\psi_{n g}$ denotes the free energy of two surface layers atoms, $\psi_{n g 1}$ denotes the free energy of two next surface layers atoms and denotes the free energy of the $\left(n^{*}-4\right)$ internal layers atoms. The number of internal layers atoms, next surface layers atoms, surface layers atoms are, respectively

$$
\begin{gathered}
N_{t r}=\left(n^{*}-4\right) N_{L}=\left(\frac{N}{N_{L}}-4\right) N_{L}=N-4 N_{L}, \\
N_{n g 1}=2 N_{L}=N-\left(n^{*}-2\right) N_{L} ; N_{n g}=2 N_{L}=N-\left(n^{*}-2\right) N_{L} .
\end{gathered}
$$

The free energy of the system and of an atom is given by

$$
\psi=N_{t r} \psi_{t r}+N_{n g 1} \psi_{n g 1}+N_{n g} \psi_{n g}=\left(N-4 N_{L}\right) \psi_{t r}+2 N_{L} \psi_{n g 1}+2 N_{L} \psi_{n g} .
$$




$$
\frac{\psi}{N}=\left[1-\frac{4}{n^{*}}\right] \psi_{t r}+\frac{2}{n^{*}} \psi_{n g 1}+\frac{2}{n^{*}} \psi_{n g} .
$$

Using that $\bar{a}$ is the average nearest-neighbor distance, $\bar{b}$ is the average thickness of twolayers and $\bar{a}_{c}$ is the average lattice constant respectively, we can easily calculated the relation

$$
\bar{b}=\frac{\bar{a}}{\sqrt{2}} ; \bar{a}_{c}=2 \bar{b}=\sqrt{2} \bar{a} .
$$

The thickness $d$ of thin film can be given by

$$
\begin{aligned}
d & =2 b_{n g}+2 b_{n g 1}+\left(n^{*}-5\right) b_{t r}=\left(n^{*}-1\right) \bar{b} \\
& =\sqrt{2} a_{n g}+\sqrt{2} a_{n g 1}+\frac{n^{*}-5}{\sqrt{2}} a_{t r}=\left(n^{*}-1\right) \frac{\bar{a}}{\sqrt{2}} .
\end{aligned}
$$

In Eq. (21), the average nearest-neighbor of surface layers atoms, next surface layers atoms and internal layers atoms in metal thin film at a given temperature $T$ can be determined as

$$
a_{n g}=a_{n g}(0)+<u_{i}^{n g}>_{0}, a_{n g 1}=a_{n g 1}(0)+<u_{i}^{n g 1}>_{0}, a_{t r}=a_{t r}(0)+<u_{i}^{t r}>_{0},
$$

where $a_{n g}(0) ; a_{n g 1}(0) ; a_{t r}(0)$ denotes the nearest-neighbor at zero temperature of surface layers atoms, next surface layers atoms, internal layers atoms, respectively, which can be determined from experiment or from the minimum condition of the potential energy of the system. The average nearest-neighbor of thin film is determined as

$$
\bar{a}=\frac{2 a_{n g}+2 a_{n g 1}+\left(n^{*}-5\right) a_{t r}}{n^{*}-1} .
$$

Finally, we obtain the expression of the free energy through the number of layers as follows

$$
\frac{\psi}{N}=\frac{d \sqrt{2}-3 \bar{a}}{d \sqrt{2}+\bar{a}} \psi_{t r}+\frac{2 \bar{a}}{d \sqrt{2}+\bar{a}} \psi_{n g}+\frac{2 \bar{a}}{d \sqrt{2}+\bar{a}} \psi_{n g 1} .
$$

\section{II.3. Thermodynamic quantities of the metal thin film}

The average thermal expansion coefficient of metal thin film can be calculated as follows

$$
\alpha=\frac{K_{B}}{\bar{a}} \frac{d \bar{a}}{d \theta}=\frac{d_{n g} \alpha_{n g}+d_{n g 1} \alpha_{n g 1}+\left(d-d_{n g}-d_{n g 1}\right) \alpha_{t r}}{d},
$$

with

$$
\alpha_{n g}=\frac{k_{B}}{a_{n g}} \frac{\partial\left\langle u_{i}^{n g}\right\rangle_{0}}{\partial \theta} ; \alpha_{n g 1}=\frac{k_{B}}{a_{n g 1}} \frac{\partial\left\langle u_{i}^{n g 1}\right\rangle_{0}}{\partial \theta} ; \alpha_{t r}=\frac{k_{B}}{a_{t r}} \frac{\partial\left\langle u_{i}^{t r}\right\rangle_{0}}{\partial \theta},
$$

here, $d_{n g}, d_{n g 1}$ are the surface layers and next surface layers thickness, respectively. With the aid of the free energy formula $\Psi=E-T S$, we can find the thermodynamic quantities of the system.

The specific heats at constant volume $C_{V}$ is $[9,10]$

$$
C_{V}=\left[\frac{\partial E}{\partial T}\right]_{V}=-T \frac{\partial^{2} \psi}{\partial T^{2}}=\frac{d \sqrt{2}-3 \bar{a}}{d \sqrt{2}+\bar{a}} C_{V}^{t r}+\frac{2 \bar{a}}{d \sqrt{2}+\bar{a}} C_{V}^{n g}+\frac{2 \bar{a}}{d \sqrt{2}+\bar{a}} C_{V}^{n g 1}
$$


where

$$
\begin{aligned}
& C_{V}^{t r}=3 k_{B} N\left\{\frac{x_{t r}^{2}}{\sinh ^{2} x_{t r}}+\frac{2 \theta}{k_{t r}^{2}}\left[\left(2 \gamma_{2 t r}+\frac{\gamma_{1 t r}}{3}\right) \frac{x_{t r}^{3} \operatorname{coth} x_{t r}}{\sinh ^{2} x_{t r}}+\frac{2 \gamma_{1 t r}}{3}\right.\right. \\
& \left.\left.-\gamma_{2 t r}\left(\frac{x_{t r}^{4}}{\sinh ^{4} x_{t r}}+2 \frac{x_{t r}^{4} \operatorname{coth}^{2} x_{t r}}{\sinh ^{2} x_{t r}}\right)\right]\right\}, \\
& C_{V}^{n g}=3 k_{B} N\left\{\frac{x_{n g}^{2}}{\sinh ^{2} x_{n g}}+\frac{2 \theta}{k_{n g}^{2}}\left[\left(2 \gamma_{2 n g}+\frac{\gamma_{1 n g}}{3}\right) \frac{x_{n g}^{3} \operatorname{coth} x_{n g}}{\sinh ^{2} x_{n g}}+\frac{2 \gamma_{1 n g}}{3}\right.\right. \\
& \left.\left.-\gamma_{2 n g}\left(\frac{x_{n g}^{4}}{\sinh ^{4} x_{n g}}+2 \frac{x_{n g}^{4} \operatorname{coth}^{2} x_{n g}}{\sinh ^{2} x_{n g}}\right)\right]\right\}, \\
& C_{V}^{n g 1}=3 k_{B} N\left\{\frac{x_{n g 1}^{2}}{\sinh ^{2} x_{n g 1}}+\frac{2 \theta}{k_{n g 1}^{2}}\left[\left(2 \gamma_{2 n g 1}+\frac{\gamma_{1 n g 1}}{3}\right) \frac{x_{n g 1}^{3} \operatorname{coth} x_{n g 1}}{\sinh ^{2} x_{n g 1}}+\frac{2 \gamma_{1 n g 1}}{3}\right.\right. \\
& \left.\left.-\gamma_{2 n g 1}\left(\frac{x_{n g 1}^{4}}{\sinh ^{4} x_{n g 1}}+2 \frac{x_{n g 1}^{4} \operatorname{coth}^{2} x_{n g 1}}{\sinh ^{2} x_{n g 1}}\right)\right]\right\} .
\end{aligned}
$$

The specific heats at constant presure $C_{p}$ is $[9,10]$

$$
C_{P}=C_{V}-T\left(\frac{\partial V}{\partial T}\right)_{P}^{2}\left(\frac{\partial P}{\partial V}\right)_{T}=C_{V}+9 T V \alpha^{2} B_{T}
$$

\section{NUMERICAL RESULTS AND DISCUSSIONS}

The expressions derived in previous section have been used to evaluate the thermodynamic properties of the metals thin film with face-centered cubic structure for $\mathrm{Al}, \mathrm{Au}$ and Ag. We used the Lennard-Jones potential with the potential parameters $D, m, n$ and $r_{0}$ in Table 1

$$
\varphi(r)=\frac{D}{(n-m)}\left[m\left(\frac{r_{0}}{r}\right)^{n}-n\left(\frac{r_{0}}{r}\right)^{m}\right] .
$$

Using the effective pair potential of Eq. (32) and Eq. (15), it is straightforward to get the interaction energy $u_{0}^{n g}$, the quantity $k_{n g}$, and the parameter $\gamma_{n g}$, in the crystal as

$$
\begin{gathered}
u_{0}^{n g}=\frac{D}{(n-m)}\left[m A_{n}\left(\frac{r_{0}}{a_{n g}}\right)^{n}-n A_{m}\left(\frac{r_{0}}{a_{n g}}\right)^{m}\right], \\
k_{n g}=\frac{D m n}{2(n-m) a_{n g}^{2}}\left\{\left[(n+2) A_{n g, n+4}^{a_{n g, i x}^{2}}-A_{n g, n+2}\right]\left(\frac{r_{0}}{a_{n g}}\right)^{n}\right. \\
\left.-\left[(m+2) A_{n g, m+4}^{a_{n g, i x}^{2}}-A_{n g, m+2}\right]\left(\frac{r_{0}}{a_{n g}}\right)^{m}\right\}, \\
\gamma_{1 n g}=\frac{1}{48} \frac{D m n}{(n-m) a_{n g}^{4}}\left[\begin{array}{l}
(n+2)(n+4)(n+6) A_{n g, n+8}^{a_{n g, i x}^{4}} \\
-6(n+2)(n+4) A_{n g, n+6}^{a_{n g, i x}^{2}}+3(n+2) A_{n g, n+4}
\end{array}\right]\left(\frac{r_{0}}{a_{n g}}\right)^{n} \\
-\frac{1}{48} \frac{D m n}{(n-m) a_{n g}^{4}}\left[\begin{array}{l}
(m+2)(m+4)(m+6) A_{n g, n+8}^{a_{n g}^{4}, i x} \\
-6(m+2)(m+4) A_{n g, n+6}^{a_{n g}^{2}, i x}+3(n+2) A_{n g, n+4}
\end{array}\right]\left(\frac{r_{0}}{a_{n g}}\right)^{m},
\end{gathered}
$$




$$
\begin{aligned}
& \gamma_{2 n g}=\frac{1}{8} \frac{D m n}{(n-m) a_{n g}^{4}}\left[\begin{array}{l}
(n+2)(n+4)(n+6) A_{n g, n+8}^{a_{n g, i x}^{2} a_{n g, i y}^{2}} \\
-2(n+2)(n+4) A_{n g, n+6}^{a_{n g, i x}^{2}}+(n+2) A_{n g, n+4}
\end{array}\right]\left(\frac{r_{0}}{a_{n g}}\right)^{n} \\
& -\frac{1}{8} \frac{D m n}{(n-m) a_{n g}^{4}}\left[\begin{array}{l}
(m+2)(m+4)(m+6) A_{n g, n+8}^{a_{n g, i x}^{2} a_{n g, i y}^{2}} \\
-2(m+2)(m+4) A_{n g, m+6}^{a_{n g, i x}^{2}}+(n+2) A_{n g, m+4}
\end{array}\right]\left(\frac{r_{0}}{a_{n g}}\right)^{m}, \\
& \gamma_{n g}=\frac{1}{12} \frac{D m n}{(n-m) a_{n g}^{4}}\left[\begin{array}{c}
(n+2)(n+4)(n+6)\left(A_{n g, n+8}^{a_{n g, i x}^{4}}+6 A_{n g, n+8}^{a_{n g, i x}^{2} a_{n g, i y}^{2}}\right) \\
-18(n+2)(n+4) A_{n g, n+6}^{a_{n g, i x}^{2}}+9(n+2) A_{n g, n+4}
\end{array}\right]\left(\frac{r_{0}}{a_{n g}}\right)^{n} \\
& -\frac{1}{12} \frac{D m n}{(n-m) a_{n g}^{4}}\left[\begin{array}{c}
(m+2)(m+4)(m+6)\left(A_{n g, n+8}^{a_{n g, i x}^{4}}+6 A_{n g, n+8}^{a_{n g, i x}^{2} a_{n g, i y}^{2}}\right) \\
-18(m+2)(m+4) A_{n g, n+6}^{a_{n g, i x}^{2}}+9(m+2) A_{n g, n+4}
\end{array}\right]\left(\frac{r_{0}}{a_{n g}}\right)^{m},
\end{aligned}
$$

where $A_{n g, n}, A_{n g, m}, A_{n g, n}^{a_{n g, i x}^{2}}, A_{n g, m}^{a_{n g, i x}^{2}}, A_{n g, n}^{a_{n g, i x}^{4}}, A_{n g, m}^{a_{n g, i x}^{4}}, A_{n g, n}^{a_{n g, i x}^{2} a_{n g, i y}^{2}}, A_{n g, m}^{a_{n g, i x}^{2} a_{n g, i y}^{2}}$ are the structural sums for the given crystal.

A similar derivation can be also done for next surface layers atoms and internal layers atoms of the system, we obtained values for $u_{0}^{n g 1}, k_{n g 1}, \gamma_{1 n g 1}, \gamma_{2 n g 1}, \gamma_{n g 1}, u_{0}^{t r}, k_{t r}, \gamma_{1 t r}, \gamma_{2 t r}, \gamma_{t r}$.

With the use of the expressions obtained in Sec. II, we calculate the values of the everage lattice constant, $\bar{a}_{c}$, the thickness, $d$, and thermodynamic properties: the specific heats at constant volume and constant pressure, $C_{V}$ and $C_{p}$, and the linear thermal expansion coefficients, $\alpha$ for $\mathrm{Au}, \mathrm{Ag}$ and $\mathrm{Al}$ metals thin film at various temperatures. The calculated results are presented in Figs. 2-7.

Table 1. Lennard-Jones potential parameters for $\mathrm{Au}, \mathrm{Ag}$ and $\mathrm{Al}$ of metals thin film [11]

\begin{tabular}{|l|l|l|l|l|}
\hline Metals & $\mathrm{n}$ & $\mathrm{m}$ & $r_{0},\left(A^{0}\right)$ & $D / k_{B},(K)$ \\
\hline$A u$ & 10.5 & 5.5 & 2.8751 & 4683.0 \\
\hline$A g$ & 11.5 & 5.5 & 2.8760 & 3325.6 \\
\hline$A l$ & 12.5 & 5.5 & 2.8541 & 2995.6 \\
\hline
\end{tabular}

In Figs. 2-3, we present the average lattice constant of Au metal thin film as a function of the thickness. It has been compared with those of gold sputtered on glass presented in [2]. We obtained results of average lattice constants for Ag and Al decrease also with increasing thickness. These results are in agreement with conclusions by Dibyendu Hazra [12]. Furthermore, we realize that: $(i)$ The average lattice constant depends on the changing of absolute temperature and the thickness. At very low temperatures $T<90 K$, the average atomic displacements at the surface layers and next surface layers are very small. It leads to atomic distances at the internal layers are larger than those at the surface layers of the thin film, so the average lattice constant increasing with the thickness. When temperature increases, in temperature range $100 K<T<700 K$, the link forces acting on atoms at surface layers and next surface layers are smaller than those at internal layers (here we have considered to the surface effect - the defect atoms on surface layers and next surface layers of the thin films). The surface layers and next surface layers atoms 


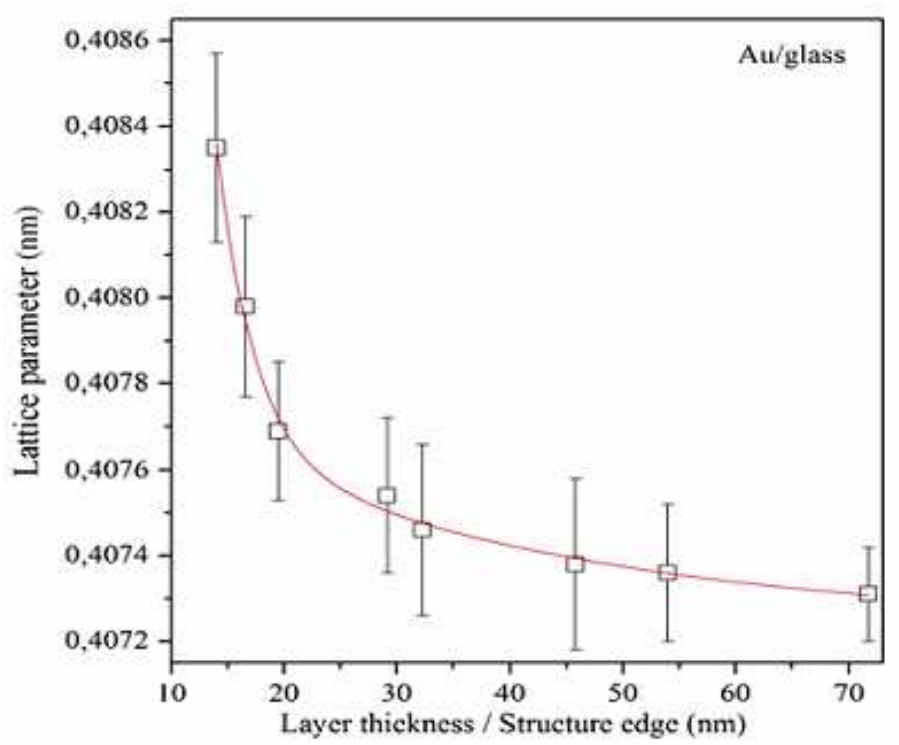

Fig. 2. Dependence of Au lattice parameter on structure edge or thickness of Au on glass.

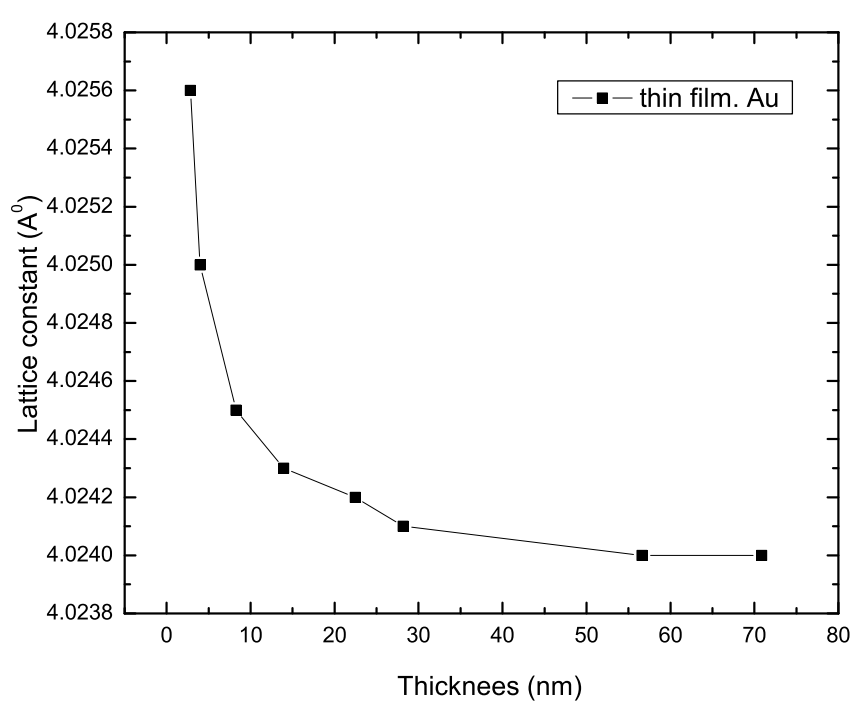

Fig. 3. Dependence on thicknees of the lattice constant for $\mathrm{Au}$ at $T=300 \mathrm{~K}$.

fluctuate more strongly than the internal layers atoms leading to the atom-atom distance at external layers is larger than that of the internal layers. Therefore, the average lattice constants decrease with the increasing of thickness. These results are in agreement with the laws of other authors $[2,12]$. (ii) When the thickness increases, the average lattice constants approach the bulk value. 


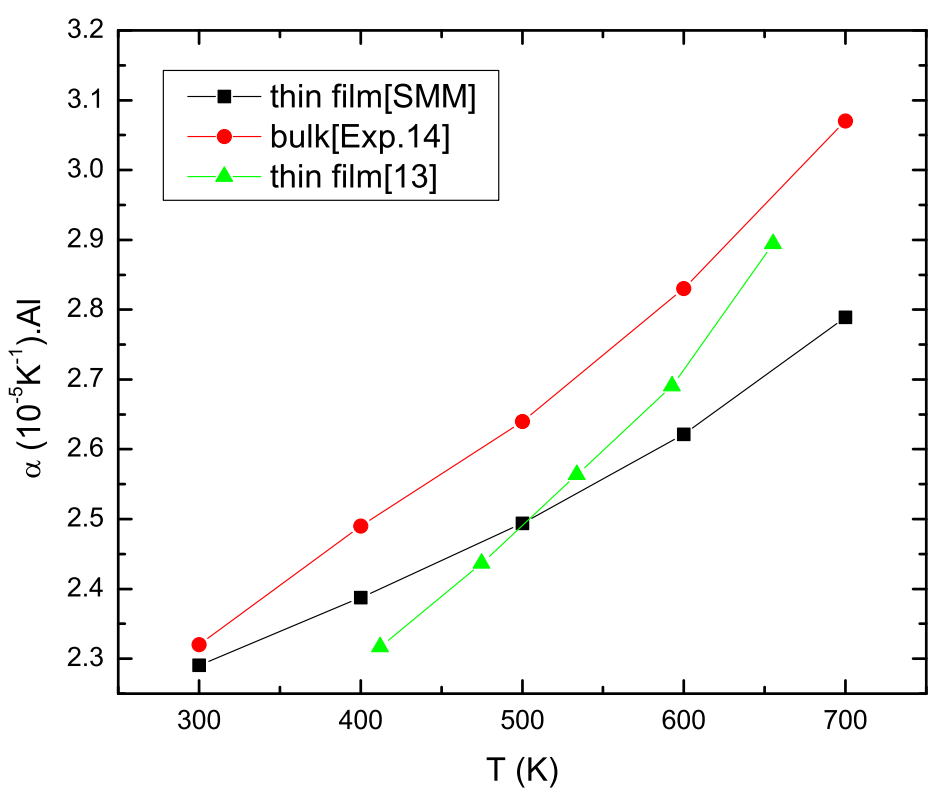

Fig. 4. Temperature-dependent thermal expansion coefficients of Al.

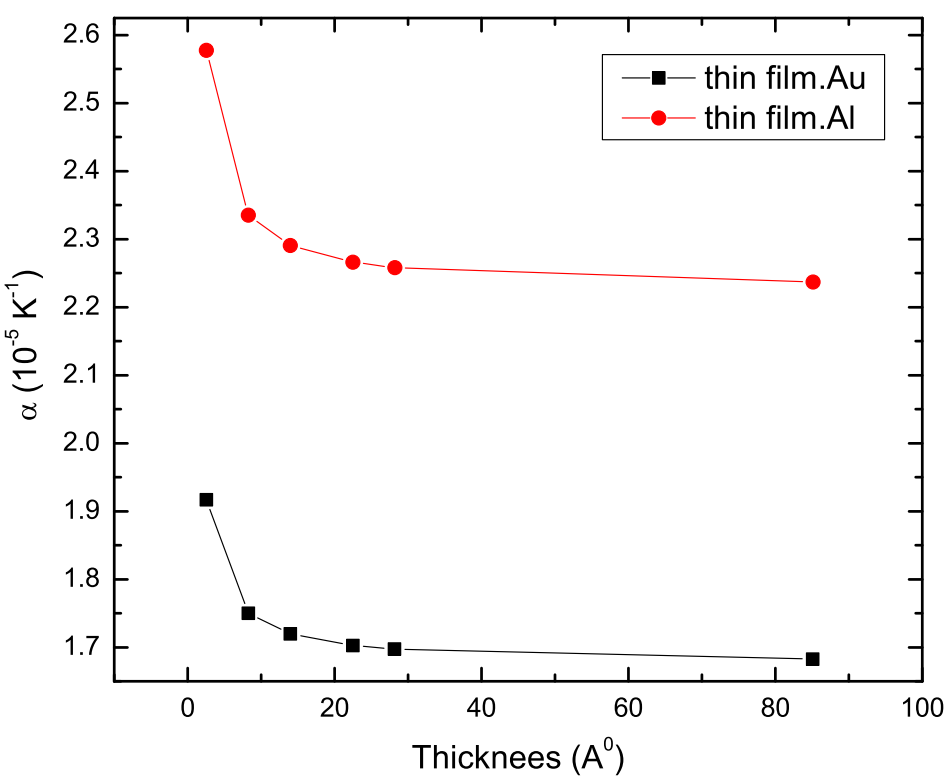

Fig. 5. Thickness-dependent thermal expansion coefficients at $T=300 \mathrm{~K}$.

In Fig. 4, we present the temperature dependence of the thermal expansion coefficients of metal thin film for Al. It can be seen that the thermal expansion coefficients 
increase with absolute temperature $T$. Our calculation results have been compared with the results presented in [13] for Al thin film, and Al bulk [14] showing the good agreement. In Fig. 5, we also show the thickness dependence of the thermal expansion coefficients of thin film $\mathrm{Au}$ and $\mathrm{Al}$. Our calculation results show that the thermal expansion coefficients for $\mathrm{Au}$ and $\mathrm{Al}$ decrease with increasing thickness. When the thickness increases then the thermal expansion coefficients approach the bulk value in agreement with the results presented in [14]. We also realize that the thermal expansion coefficients of metal thin film change with the fluctuation of absolute temperature and the thickness. In temperature range $100 K<T<700 K$, from Eq. (25), it can be seen that, the average thermal expansion coefficients decrease with the increasing of thickness. Our calculations results shown that the thermal expansion coefficients increase with absolute temperature $T$. These results are in good agreement with the results presented by O. Kraft and W. D. Nixx in [15].

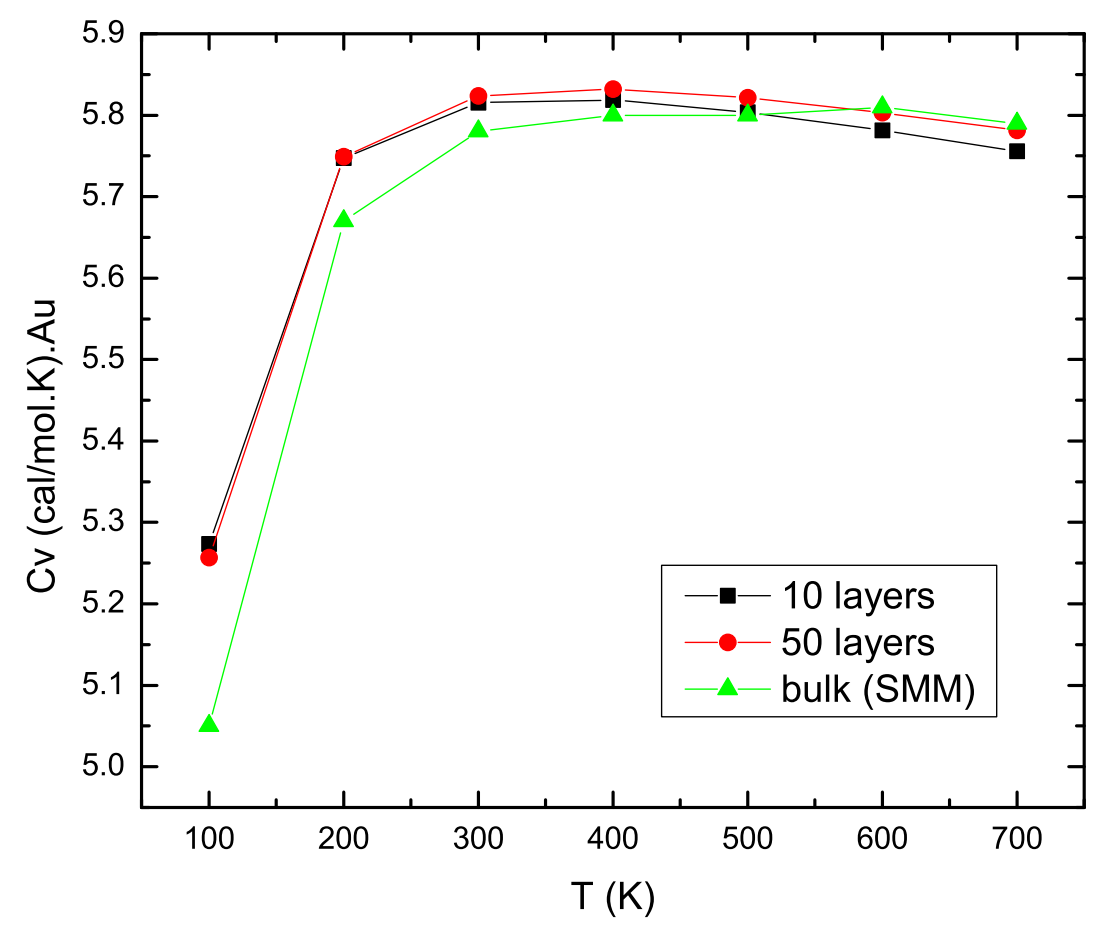

Fig. 6. Temperature-dependent specific heats at constant volume of Au.

In Figs. 6-7, we present the temperature dependence of the specific heats at constant volume and of the specific heats at constant pressure of metal thin film. At low temperature $T<300 K$, the specific heats at constant volume and the specific heats at constant pressure increase with the absolute temperature. At high temperatures, the volumetric heat capacities decrease less with the absolute temperature and reverse the 


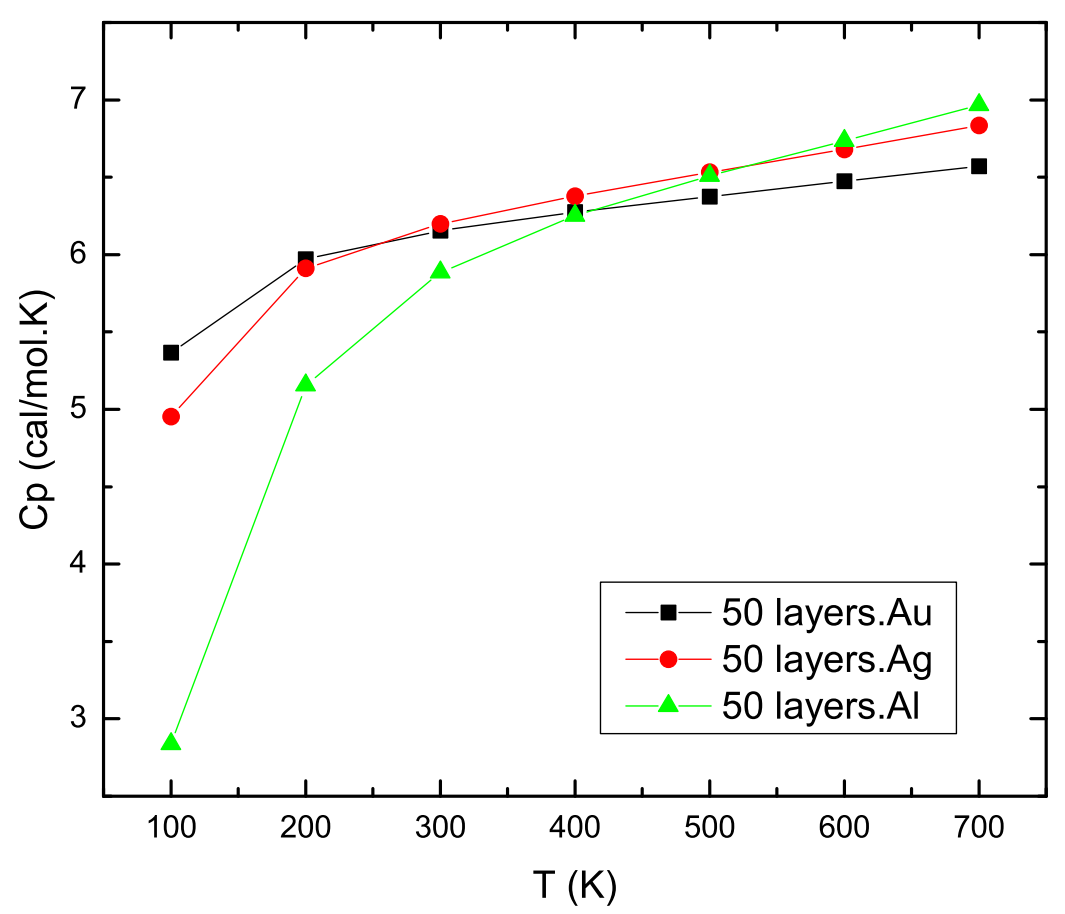

Fig. 7. Temperature-dependent specific heats at constant pressure for 50 layers.

pressure-specific heat capacities increase less with the absolute temperature. The rise and fall of the specific heats indicate the stronger anharmonicity contribution of the thermal lattice vibration at high temperature.

\section{CONCLUSIONS}

We have presented the $S M M$ formalism by using the Lennard-Jones interaction potentials, and investigated the thermodynamic properties of the $\mathrm{Au}, \mathrm{Ag}$ and $\mathrm{Al}$ metals thin film. The $S M M$ is simple and physically transparent, and thermodynamic quantities of metals thin film with FCC structures can be expressed in closed forms within the fourth order moment approximation of the atomic displacements. In general, we have obtained good agreement in the thermodynamic quantities between our theoretical calculations and other theoretical results, and experimental values.

\section{ACKNOWLEDGEMENTS}

This research is funded by Vietnam National Foundation for Science and Technology Development (NAFOSTED) under grant number 103.01 - 2011.16 


\section{REFERENCES}

[1] L. H. Liang and B. Li, Phys. Rev. B, 73(15) (2006), 153303

[2] Z. Kolska et al., Materials Letters, 64 (2010), 1160-1162

[3] L. H. Liang et al., Solid State Communications, 121(8) (2002), 453-455

[4] S. Nakao et al., J. Micromech Microeng, 16 (2006), 715

[5] E. Bonderover and S. Wagner, JEEE Elektron Dev Lett, 25 (2004), 295

[6] E. Roduner, Chem Soc Rev, 35 (2006), 583

[7] S. Kanagaraj and S. Pattanayak, Cryogenics, 43 (2003), 399-424

[8] A. Biswas et al., Vac Technol Coat, 7 (2006), 57

[9] K. Masuda-Jindo et al., Phys. Rev. B, 67 (2003), 094301

[10] N. Tang, V. V. Hung, Phys. Stat. Sol(b), 149 (1998), 511-519

[11] M. N. Magomedov, J. Fiz. Khimic, 61 (1987), 1003

[12] D. Hazra et al., J. Appl. Phys., 103 (2008), 103535

[13] G. Cornella et al., MRS Online Proc. Lib., 505 (1998), 527-532

[14] R.O. Simmons and R.W. Balluffi, Phys. Rev., 117 (1960), 52

[15] O. Kraft and W. D. Nix., J. Appl. Phys., 83(1998), 3035-3038 